\title{
Comparisons of compression characteristics in compression forming of porous cylinder based on slab method and finite element method under constant shear friction
}

\author{
Gow-Yi Tzou ${ }^{1, *}$, Yeong-Maw Hwang ${ }^{2}$, and Sai-Chih Pan $^{2}$ \\ ${ }^{1}$ Department of Mechanical and Automation Engineering, Chung Chou University of Science and Technology, Taiwan, R.O.C. \\ ${ }^{2}$ Department of Mechanical and Electro-Mechanical Engineering, National Sun Yat-Sen University, Taiwan, R.O.C.
}

\begin{abstract}
The study assumes constant shear friction to perform mechanics analysis and comparisons of compression characteristics in compression forming of porous cylinder based on slab method and finite element method. The vertical stress $(p)$, radial stress $(q)$, compression force $(P)$, average radius, and density after forming can be systematically explored. Because the volume in forming of porous cylinder cannot keep constant, just only keep the mass constancy. Therefore, the derivation is more complicated. The mechanics analytical model established by the slab method compares with the FEM using DEFORM-2D analysis software each other to realize the variations of both models; the maximum average error for the compressive load $(P)$ under various reduction ratios is $14.8 \%$ between the slab method and the finite element method. The results with a good agreement are shown in the study. These models can be offered to the reference of porous cylinder compression in industries.
\end{abstract}

\section{Introduction}

The porous materials have a wide range of applications in the diverse industries. They are important materials for lubricating bearings, metal filters, metal electrodes, and light-weight parts for the automotive industry. There have been many relevant studies discussed in several aspects.

Yang et al. [1] explored the porous materials on the relationship of bulging deformation, forging force and density change during open die forging. By FEM analysis and other experiment results, Yang et al. [1] investigated the applicability of FEM simulations as well. Lee et al. [2] emphasized the importance of the empty holes effect and the density distribution in the study of porous materials. In addition to their criteria on plastic yielding, they also compared with other criteria on yielding in various studies. Based on their yielding condition, Gurson, Shirizly et al. [3] used experiments in macro and micro scales to prove the accuracy of FEM in predicting the density of porous materials during compression processing. Doege et al. [4] predicted the density variations of the porous metal ring in the compacting process, and proposed an analytic method of ordinary differential equations (ODE), which is easier than Hill's partial differential equations (PDE). As for the yielding criteria and the plastic behaviour [5-9], Huang et al. [5] used the sintered powder compacts to perform uniaxial tension and compression tests under various frictional conditions, and the results were used to compare to FEM each other. They described the plastic behavior of porous materials in Gurson-Tvergaard's criterion on yielding. Green [6] used rigorous mathematics to establish the plasticity theory for porous solids, and proposed the differential equations and the relationship of yield criterion on the stress-strain and the void ratio. This was the basis for the derivation of the slab method which is proposed in this paper. Using the stress-strain curve data in the sintered copper experiment, Shima et al. [7] proposed the plasticity theory for porous metals and applied it to a friction-free closed-dies compression experiment to verify its practicability. Doraivelu et al. [8] reviewed many yield criteria proposed by various scholars. They aimed at the compressible powder metallurgy materials to propose a new yielding function, and used aluminum alloy (A7091) uniaxial compression experiment to test the accuracy and practicability. Dias et al. [9] used the plasticity equation to analyze the parameters of the sintered porous materials after the forging process. They confirmed the experiment results agreeable to the theoretical analysis of the proposed model, which can be applied to the cold forging of sintered performs. Jha et al. [10] detailed the experiments on forming characteristics and failure mechanisms from the start of spraying iron metal powder to the completion of sintering processing. In the forming properties, they discussed the size of powder particles in details, and also classified the types of crack to the failure mechanisms. Nguyen et al. [11] investigated the mechanical behaviour of porous functionally graded (FG) beams; they indicated that it is necessary to take the effect of porosities into consideration in the vibration analysis of FG beams traversed by moving forces.

\footnotetext{
Corresponding author: johnsontzou@gmail.com
} 


\section{Simulation analysis and modelling}

\subsection{Finite element method}

In this study, DEFORM-2D is used to perform the simulation analysis on the compression forming of porous cylinder. Based on the finite element method, the DEFORM-2D analyzes two-dimensional flow in the process of metal forming. It is often used to analyze of forging, extrusion, drawing, upset forging etc. in forming process used by industrial and academic groups. The software settings in DEFORM-2D include: porous materials; the function of flow stress, $\sigma=K \varepsilon^{n}$ $(\mathrm{K}=500 \mathrm{MPa}, \mathrm{n}=0.3)$; Young's modulus, $\mathrm{E}=210 \mathrm{GPa}$; Cylindrical height to diameter ratio (Hi/Di) $15 / 12=1.25$, $12 / 12=1,9 / 12=0.75$; initial relative density before compression, $\rho_{\mathrm{i}}=0.7$; numbers of element $=3000$; constant shear friction factor between workpieces and dies (m) are taken as $0.1,0.3,0.5$; compression speed of upper die $=1 \mathrm{~mm} / \mathrm{s}$.

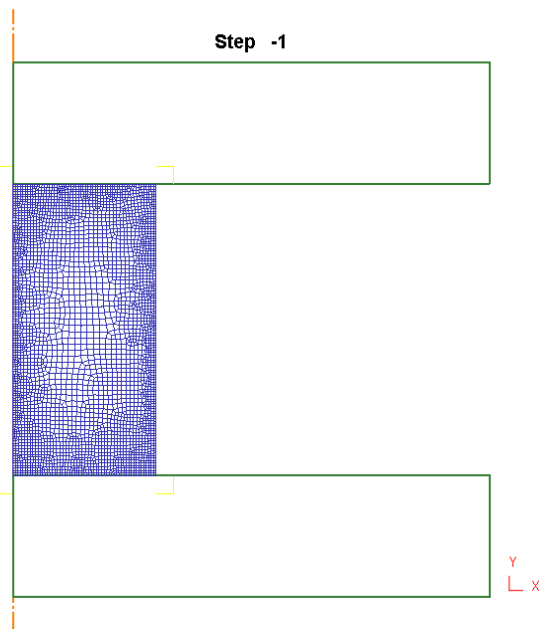

Fig. 1. The diagram of pre-process in compression forming of porous cylinder in DEFORM-2D.

\subsection{Slab method}

In general, before the processing effects are unknown, the slab method can analyze the influential parameters on the processing effects in advance, and probe into the conceptual discussion. By the slab method, the stress distribution and compressive force of the porous cylindrical material in compression forming are explored and then compare to the simulation results of DEFORM2D.

To simplify the complexity of derivations, the study establishes a mechanics analytical model for the porous cylinder material between upper and lower die, and assumes that the friction between the interface of the dies and cylinder is constant shear friction. With the cylinder material is porous, the compressive pressure distribution and compressive force of the porous cylinder material during the compression processing are deduced by the theory of slab method.

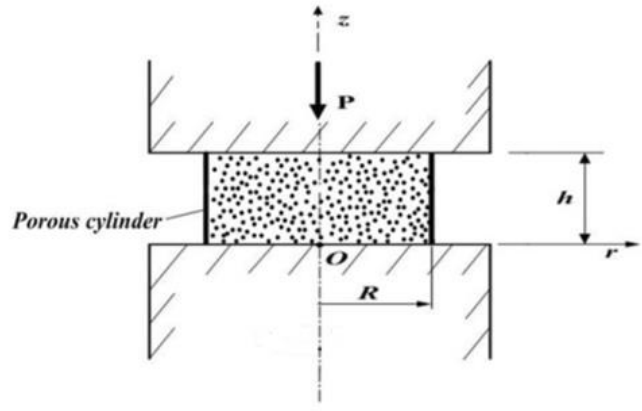

Fig. 2. Schematic diagram of porous cylinder in compression.

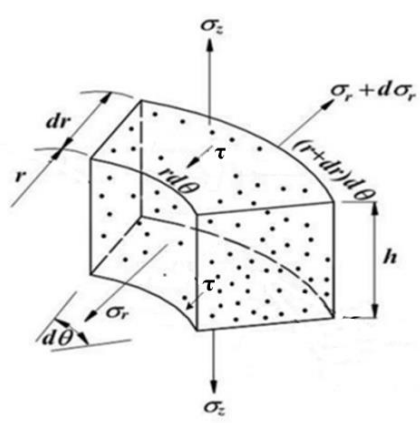

Fig. 3. Stress element diagram of porous cylinder in compression.

In order to derive the compression forming analytical model, the following basic assumptions are required:

(1)Assuming that the cylinder is a porous material, and the pores are evenly distributed in the cylindrical material.

(2)The mechanics analysis is based on axisymmetry.

(3)The stresses on the elements are distributed uniformly; radial $\left(\sigma_{\mathrm{r}}\right)$ and vertical $\left(\sigma_{\mathrm{z}}\right)$ stresses are considered as principal stresses.

(4)The interfacial friction between the die and the porous cylinder is assumed to be constant shear friction; $\tau$ is utilized in the radial direction of the cylinder material.

(5)The bulging effect on the free surface is ignored.

(6)Since the porous material has holes, the incremental deformation makes the material denser and the void space less. The conservation of mass can be applied to the porous material; the conservation of the volume is unable to be used.

Therefore, the yielding criterion of the porous material should be considered first before setting up the slab method for an analytical model of compression forming of porous material.

\subsubsection{Green's yielding criterion}

The Green's yielding criterion [4] is expressed as follows: 


$$
G \cdot J_{2}^{\prime}+B I_{1}^{2}=D Y_{i}^{2}=Y_{R}^{2}
$$

where $\boldsymbol{G}=\mathbf{3}, \quad \boldsymbol{J}_{2}^{\prime}$ is the second stress deviation tensor, $\boldsymbol{I}_{\boldsymbol{1}}$ is the first stress constant tensor, $\boldsymbol{D}=\rho_{r}^{5}, \rho_{r}$ is the relative density after the compression $\left(\rho_{\boldsymbol{r}}=1-f, f\right.$ is porosity rate), and $\boldsymbol{Y}_{\boldsymbol{i}}$ is the initial yield strength of porous material, $B=\left(\frac{1}{9}\right)\left(2.49\left(1-\rho_{r}\right)^{0.514}\right)^{2}$

From Eq.(1), when $\rho_{r}=1$, it indicates that the materials has no void, i.e. it is a sound material $(f=0)$; then $3 J_{2}^{\prime}=\boldsymbol{Y}_{i}^{2}$ is the yielding criterion for sound material. As $\rho_{r} \neq \mathbf{1}$, it indicates that the material is a porous material:

$$
J_{2}^{\prime}+\frac{B}{3} I_{1}^{2}=\frac{D Y_{i}^{2}}{3}=D\left(\frac{Y_{i}}{\sqrt{3}}\right)^{2}=D k_{i}^{2}=\left(\sqrt{D} k_{i}\right)^{2}=k_{e}^{2}=\frac{Y_{R}^{2}}{3}
$$

Where Eqs. (1) and (2) are the yielding criteria for porous material proposed by Doege et al. [4].

\subsubsection{Force equilibrium}

Using cylindrical coordinate system, the axisymmetric is satisfied. The radial force equilibrium equation is $\sum \boldsymbol{F}_{\boldsymbol{r}}=\boldsymbol{0}$.

$$
\frac{d \sigma_{r}}{d r}-\frac{2 \tau}{h}=0
$$

where $\tau=\boldsymbol{m} \boldsymbol{k}_{D}=\boldsymbol{m}\left(\sqrt{D} \boldsymbol{k}_{i}\right)=\boldsymbol{m} \sqrt{\rho_{r}^{5}} \boldsymbol{k}_{i}$.

Let $\sigma_{r}=\boldsymbol{q}, \sigma_{z}=\boldsymbol{p}, \sigma_{r}=\sigma_{\theta}$,

$$
\frac{d q}{d r}-\frac{2 m k_{D}}{h}=0
$$

The radial stress $(q)$ can be obtained.

$$
q=\frac{2 m k_{D}}{h} r+c
$$

where $c$ is an integral constant, it can be obtained by the boundary condition.

\subsubsection{Boundary condition}

The radial stress is zero on free surface of cylinder, that is $q=0$, at $r=R$, Substitute this boundary condition into Eq. (5); then the integral constant $\mathrm{c}$ is determined.

$$
c=-\frac{2 m k_{D}}{h} R
$$

\subsubsection{Radial stress}

The radial stress can be expressed as

$$
q=\frac{2 m k_{D}}{h}(r-R)
$$

\subsubsection{Compression stress (Vertical stress)}

Due to the axisymmetric problem, the radial stress equals to the hoop stress, i.e. $\sigma_{r}=\sigma_{\theta}$, and $\sigma_{r \theta}=\sigma_{\theta z}=\sigma_{z r}=0$,

$$
\begin{aligned}
& \text { so } \\
& \boldsymbol{J}_{2}^{\prime} \\
& \equiv \frac{\boldsymbol{1}}{\boldsymbol{6}}\left[\left(\sigma_{r}-\sigma_{\theta}\right)^{2}+\left(\sigma_{\theta}-\sigma_{z}\right)^{2}+\left(\sigma_{z}-\sigma_{r}\right)^{2}\right]+\sigma_{r \theta}^{2}+\sigma_{\theta z}^{2}+\sigma_{z r}^{2} \\
& =\frac{\boldsymbol{1}}{\boldsymbol{6}}\left[(\boldsymbol{q}+\boldsymbol{p})^{2}+(\boldsymbol{p}+\boldsymbol{q})^{2}\right]+\boldsymbol{0}^{2}+\boldsymbol{0}^{2}+\boldsymbol{0}^{2} \\
& =\frac{\boldsymbol{1}}{\mathbf{3}}(\boldsymbol{p}+\boldsymbol{q})^{2}
\end{aligned}
$$

Let $\sigma_{z}=-p$

$$
I_{1}=\sigma_{r}+\sigma_{\theta}+\sigma_{z}=2 \sigma_{r}+\sigma_{z}=2 q-p
$$

Substitute $\boldsymbol{J}_{2}^{\prime}$ and $\boldsymbol{I}_{\boldsymbol{I}}$ into Eq. (2)

$$
J_{2}^{\prime}+\frac{B}{3} I_{1}^{2}=k_{D}^{2}
$$

Obtain

$$
\frac{1}{3}(p+q)^{2}+\frac{B}{3}(2 q-p)^{2}=k_{D}^{2}
$$

Then

$$
p^{2}+\frac{2(1-2 B) q}{(1+B)} p+\frac{1}{(1+B)}\left[(1+4 B) q^{2}-3 k_{D}^{2}\right]=0
$$

It can be rearranged as

$p=a_{1} q+\sqrt{a_{4}-b_{1} q^{2}}$

where

$$
\begin{aligned}
& a_{1}=\frac{2 B-1}{1+B}, a_{2}=\left(\frac{2 B-1}{1+B}\right)^{2}, a_{3}=\frac{1+4 B}{1+B}, a_{4}=\frac{3 k_{D}{ }^{2}}{1+B} \\
& b_{1}=a_{3}-a_{2}, b_{2}=\frac{2 m k_{D}}{h}, b_{3}=\sqrt{\frac{a_{4}}{b_{1} b_{2}^{2}}}
\end{aligned}
$$

\subsubsection{Compression force}

The compression force can be found by integrating the vertical stress over the contact surface

$$
P=\int_{0}^{R} p d A=2 \pi \int_{0}^{R} p \cdot r d r
$$

After the cumbersome and complicated integration, the compression force can be expressed as

$$
P=\frac{-\pi a_{1} b_{2} R^{3}}{3}+\frac{2 \pi \sqrt{b_{1}} b_{2}}{3}\left(\left(b_{3}^{2}-R^{2}\right)^{3 / 2}-b_{3}^{3}\right)
$$




$$
+\pi R \sqrt{b_{1}} b_{2}\left(R \sqrt{b_{3}^{2}-R^{2}}+b_{3}^{2} \sin ^{-1} \frac{R}{b_{3}}\right)
$$

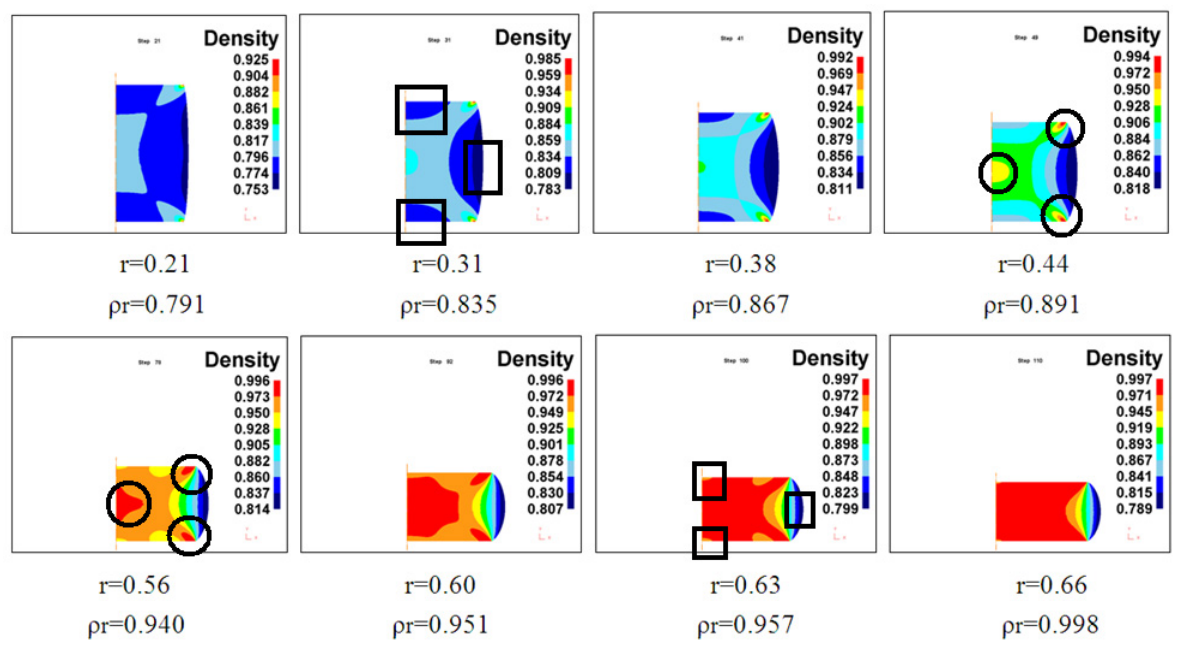

Fig. 4. The variations of relative density $\left(\rho_{\mathrm{r}}\right)$ under various reduction ratios.

\subsubsection{The mass conservation law}

The cylinder with porosity is compacted with the increase of deformation to reduce the void. Therefore, the volume constancy is not suitable for the porous material; however the mass conservation law can be satisfied. With the mass conservation law, the mass of materials before deformation is equal to one after deformation, i.e. $m_{i}=m_{r}$. As the strain in height, the initial density, and relative density of deformation are given, the deformed cylinder radius $\mathrm{R}$ can be derived from the mass conservation law as follows:

$$
R=\sqrt{\frac{\rho_{i} \cdot h_{i}}{\rho_{r} \cdot h}} R_{i}
$$

\section{Results and discussions}

Figure 4 shows the variations of relative density $\left(\rho_{\mathrm{r}}\right)$ under various reduction ratios. The reduction ratios from $21 \%$ to $66 \%$ are selected for discussions, and the relative density distributions in porous cylinder are not distributed uniformly. The areas of higher density (circled in the graphs) are located at the upper right corner, the lower right corner, and the left-middle side (i.e. peripheral on top and bottom of cylinder, and center of cylindrical axis). The areas of lower density (framed in the graphs) are located at the upper left corner, the lower left corner, and right-middle side (i.e. two ends of cylindrical axis, and the middle part of cylinder outside peripheral). The peripheral on top and bottom of the cylinder and the center of the cylinder are compactly compressed at first (i.e. $\rho_{\mathrm{r}}$ approached to the value of 1.0 rapidly); contrarily, the center on top and bottom of the cylinder and the bulging area of the cylindrical periphery are densely compressed at last (i.e. $\rho_{\mathrm{r}}$ approached to the value of 1.0 slowly).

Figure 5 shows the velocity field distributions under different reductions, it shows the flow direction of the material around the cylindrical axis is axially downward, and then flow to the radial direction. The flow rate is the highest on the cylinder top and the lowest near the cylinder bottom. The flow direction on the cylindrical periphery inclines to the right, and the radial direction will be more rightward from top to bottom.

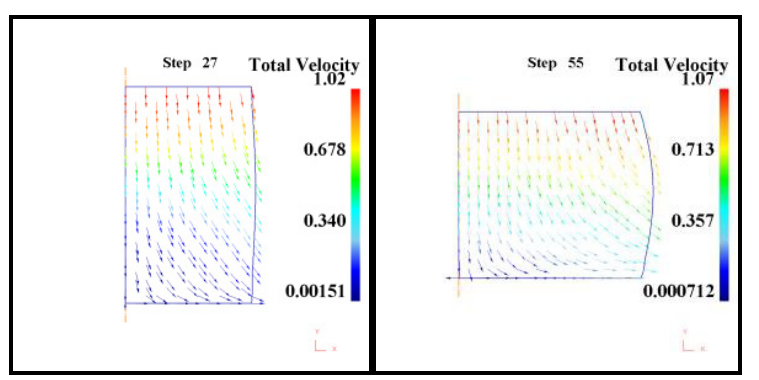

Fig. 5. The velocity field distribution under different reductions.

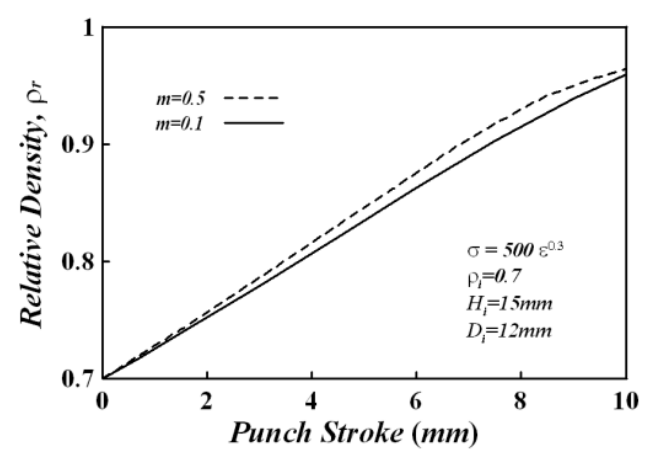

Fig. 6. Variations of the relative density with punch strokefor different frictional factors. 
Figure 6 shows variations of the relative density with punch stroke for different frictional factors. Under the same punch stroke, the greater the interface frictional factor $(\mathrm{m})$ between workpiece and die is, the higher the relative density $\left(\rho_{\mathrm{r}}\right)$ is; however, the difference is not evident. Figure 7 shows variations of the relative density with punch stroke for various aspect ratios. In the same punch stroke, the small aspect ratio corresponds to the large relative density. Namely, the compression force for a small aspect ratio will result in high relative density due to high compression force. As shown in Figure 7, the effect on the variation of aspect ratio to $\rho_{\mathrm{r}}$ is greater than on the variation of frictional factor to $\rho_{\mathrm{r}}$. In Figure 6 , as the frictional factor increases, $\rho_{\mathrm{r}}$ only changes slightly.

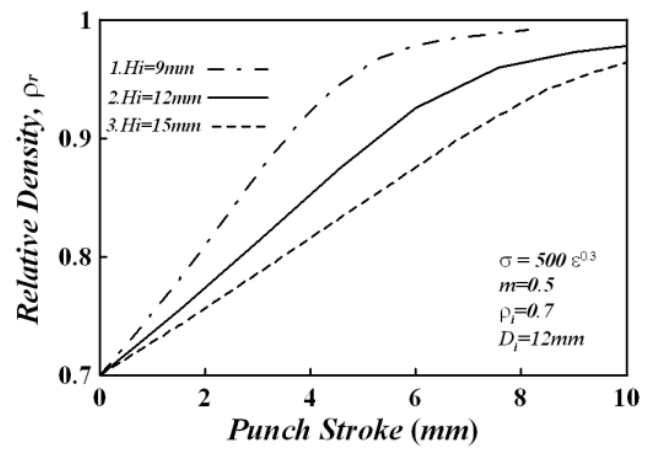

Fig. 7. Variations of the relative density with punch stroke for various aspect ratios.

From Figure 8 - 10, the distributions of vertical stress $(p)$ and radial stress $(q)$ under reduction ratio of $40 \%$ based on the slab method and the finite element method under various frictional factors $(\mathrm{m}=0.1,0.3,0.5)$. The figures show that the absolute value of vertical and radial stresses will increase when the frictional factor increases. In the figures, the curve interval is fluctuated larger when the frictional factor increased; the inaccuracy would be more within the results of two methods when the frictional factor is increased. The vertical stress $(p)$ of the finite element method is higher than that of the slab method, and the radial stress $(q)$ of the finite element method is lower than that of the slab method. As a result of increasing frictional factor, the vertical stress $(p)$ near free surface end by the finite element is lower than by the slab method.

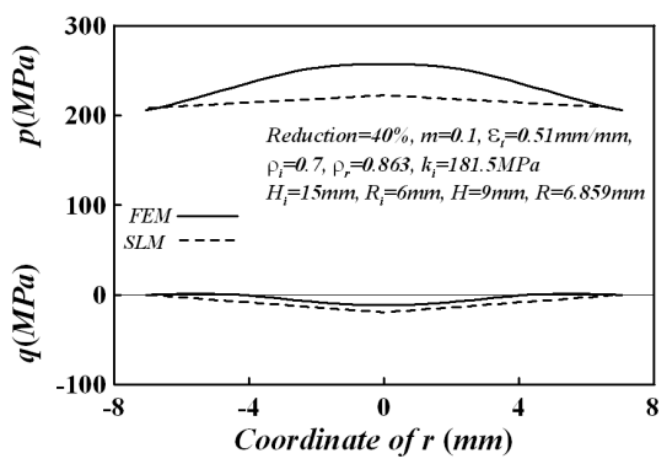

Fig. 8. Vertical stress and radial stress for $\mathrm{m}=0.1$.
Fig. 11 and 12 show the average radius under various forming conditions. Figure 11 shows that the average radius decreases when the frictional factor increases because of the higher frictional factor causing much restraint on the top and bottom of cylinder (i.e. the contact area with die); the average radius in slab method is higher than in finite element method. When the frictional factor $m=0.1$, the curves of slab method and finite element method are close (max. error is $3.24 \%$ ), and when $m=0.5$, the curve of FEM fluctuates downward largely (max. error is $6.26 \%$ ). It can be known that the slab method is not sensitive to the change of frictional factor on predicting the average radius after cylinder forming. In Figure 11, the average radius after cylinder forming decreases when the frictional factor increases.

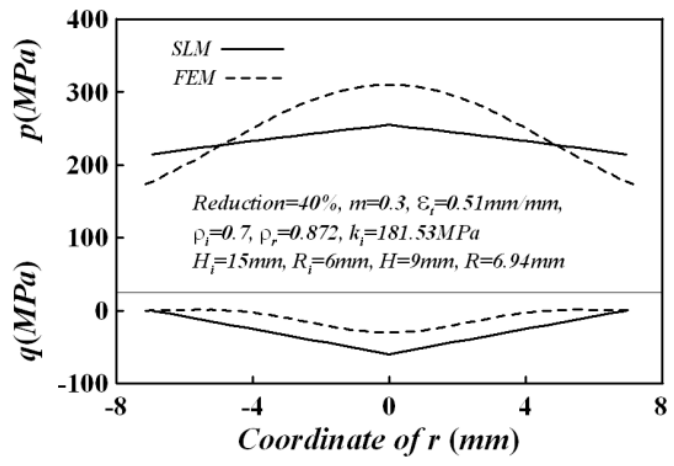

Fig. 9. Vertical stress and radial stress for $\mathrm{m}=0.3$.

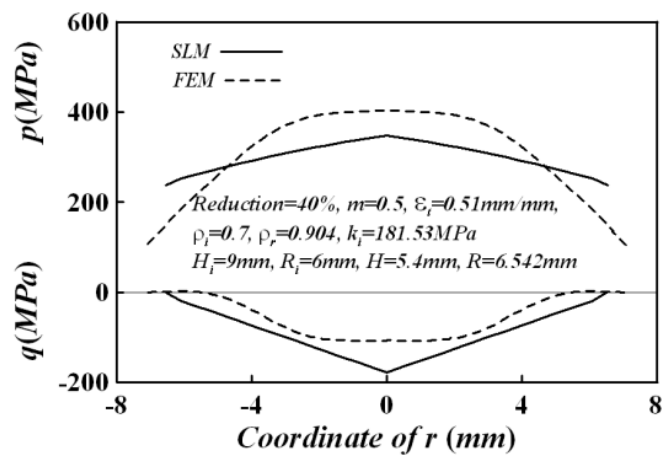

Fig. 10. Caption of the Figure 1. Below the figure.

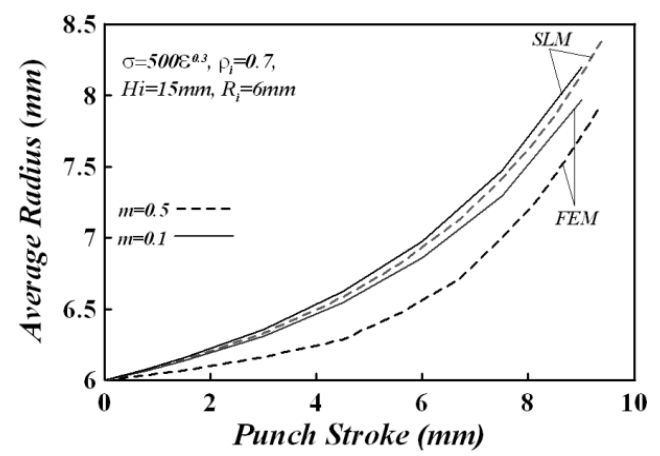

Fig. 11. Variations of average radius with punch stroke for different frictional factors. 


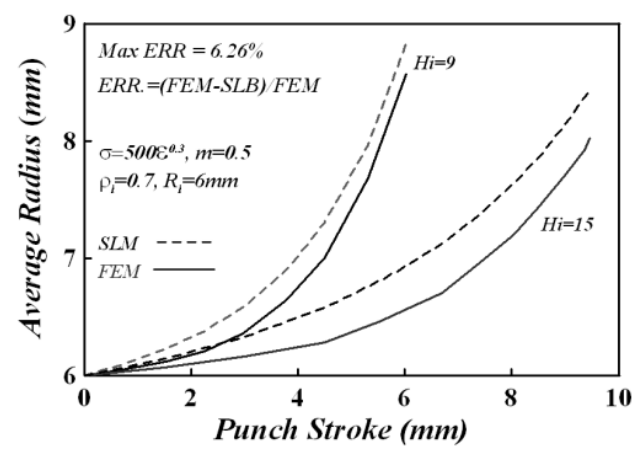

Fig. 12. Variations of average radius with punch stroke for different aspect ratios.

Because of the conservation of mass, Figure 12 shows the workpiece with lower aspect ratio in the same punch stroke has a larger average radius. It is also shown that the slab method is equally well as the finite element method on predicting the average radius after cylinder forming, and the maximum error between both methods is $6.26 \%$ only. The bulging ratio indicates the severity of cylinder bulge, and the bulging ratio is obtained by formula (13) and Fig. 13 with the radius value on upper, middle, and lower locations. The bulging ratio can be defined as below:

$$
\text { Bulging Ratio }=\left[\boldsymbol{R}_{\max }-\left(\boldsymbol{R}_{t}+\boldsymbol{R}_{b}\right) / 2\right] / \boldsymbol{R}_{i}
$$

In the finite element method, the bulging ratio can be obtained in various aspect ratios. Three different aspect ratios are compared together in Figure 14. It shows that a larger aspect ratio $(\mathrm{Hi}=15 \mathrm{~mm})$ corresponds to a higher bulging ratio; conversely, a smaller aspect ratio $(\mathrm{Hi}=9 \mathrm{~mm})$ conforms to a lower bulging ratio. That is to say a more slender workpiece after forming will have a more bulging ratio.

Figure 15 shows variations of bulging ratio with reduction for different frictional factors. The top and bottom radii of workpiece are constrained from increasing the frictional factor upon which the bulge will be intensified. The larger the frictional factor is, the greater the bulging ratios. Thus, the sufficient lubrication may decrease the bulge. Although the larger frictional factor causes more bulging, the increment of the bulge in the middle of workpiece is not more noticeable than the decrease of the radius on the contact area of die. As for the average radius, it can decrease as the frictional factor increases.

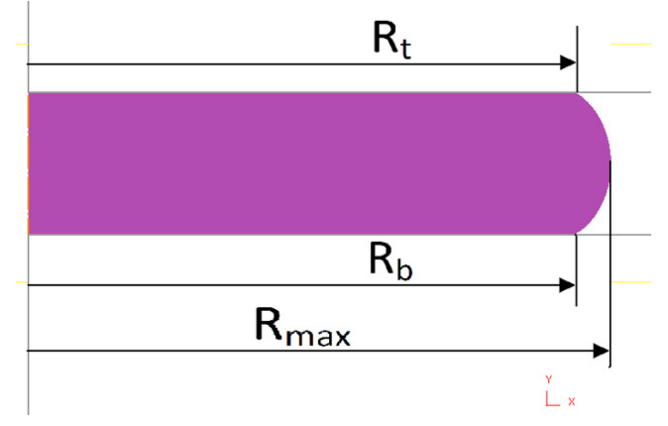

Fig. 13. Definition of bulging ratio.
Figure 16 shows variations of the compression force with punch stroke between FEM and SLM for different aspect ratios, under the same punch stroke, the maximum error between SLM and FEM is around $14.8 \%$. It is noted that the one with larger aspect ratio $(\mathrm{Hi}=15 \mathrm{~mm}$, slender) requires less compression force $(P)$, power, and energy.

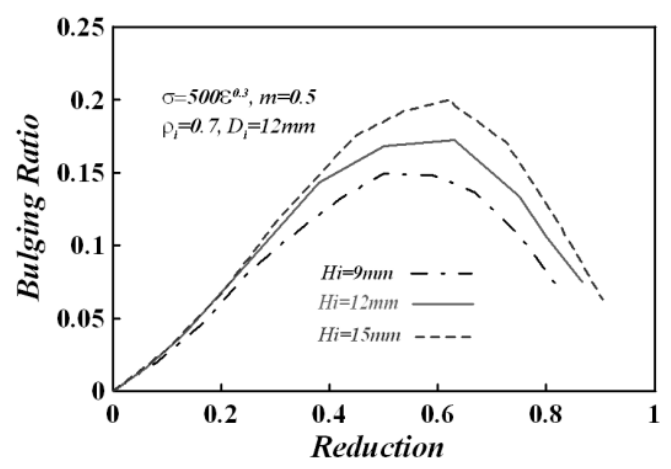

Fig. 14. Variations of bulging ratio with reduction for different aspect ratios.

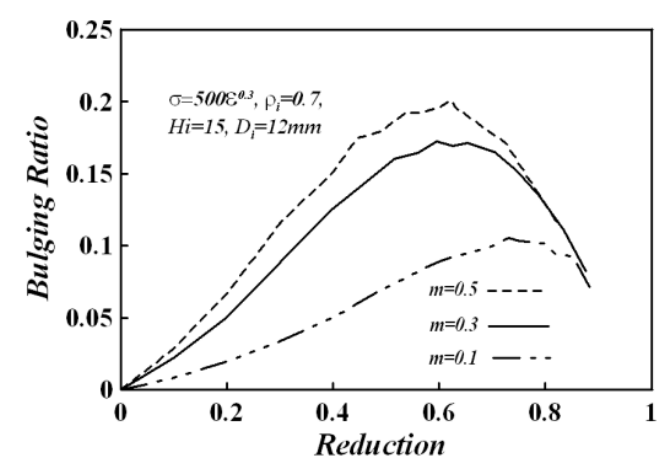

Fig. 15. Variations of bulging ratio with reduction for different frictional factors.

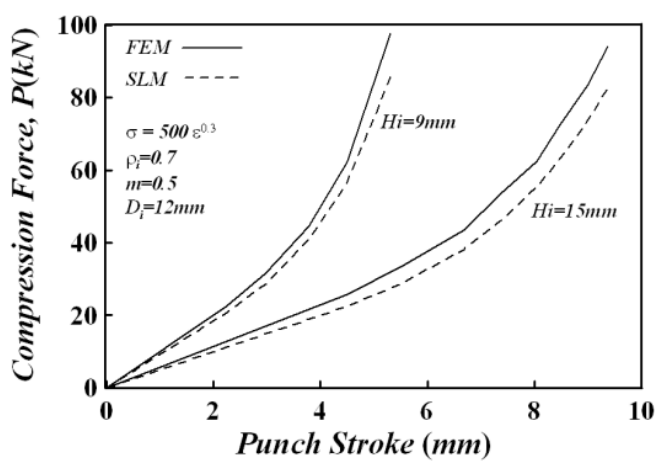

Fig. 16. Variations of the compression force with punch stroke between FEM and SLM for different aspect ratios.

Figure 17 shows variation of the compression force with punch stroke between FEM and SLM for different frictional factors. As for SLM and FEM, the larger 
interface friction $(\mathrm{m})$ causes more compression force $(P)$ under same punch stroke. In addition, FEM has a larger value compared to SLM on predicting the compression force $\left(\mathrm{P}_{\mathrm{FEM}}>\mathrm{P}_{\mathrm{SLM}}\right)$. Conversely, FEM has a smaller value than SLM on prediction the average radius (AVG $\left.\mathrm{R}_{\mathrm{FEM}}<\mathrm{AVG} \mathrm{R}_{\mathrm{SLM}}\right)$.

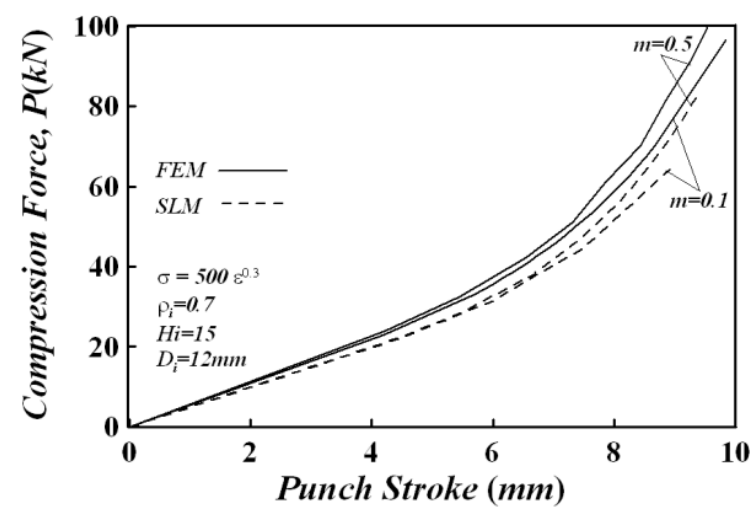

Fig. 17. Variation of the compression force with punch stroke between FEM and SLMfor different frictional factors.

\section{Conclusions}

Through a series of analyses based on the slab method and FEM simulation in this study, the results for both models are also compared to realize the variations, and the major conclusions are as:

(1)The maximum error for the compression force $(P)$ under various reduction ratios is around $14.8 \%$ between the slab method and the finite element method.

(2)The variation in relative density is not uniform during the whole compression process. The peripheral on top and bottom of the cylinder and the center of the cylinder are compressed compactly first, and the value of $\rho_{\mathrm{r}}$ will reach 1 earlier. The center on top and bottom of the cylinder and the bulging area of the cylindrical periphery are compressed slowly, and the value of $\rho_{\mathrm{r}}$ is slow to reach 1 .

(3)Since the increment of frictional factor (m) will increase the bulging rate, the sufficient lubrication will decrease the bulge. However, the increment of frictional factor increases the compression force slightly; the great difference only can be seen in a larger punch stroke.

(4)In geometric view, the larger aspect ratio causes the considerable bulge and the low compression force $\left(\rho_{\mathrm{r}}\right.$ slowly approach to 1); the compression force will be smaller under same punch stroke.

(5)By the distributions of velocity field, vertical stress, and radial stress, the major change in the compression process is the vertical direction; therefore, the radial stress $(q)$ is far less than the vertical stress $(p)$, radial velocity field is also less than axial velocity field.
(6)The relative density $\left(\rho_{\mathrm{r}}\right)$ of porous material forming will increase intensely and slow down before reaching the value of 1 .

(7)When the reduction rate is 0.66 , the relative density of cylinder is 0.998 , which is very close to the density of sound material $\left(\rho_{\mathrm{r}}=1\right)$. At the larger reduction ratio or punch stroke, the process is similar to the compression forming of sound material and produces more compression force, which is more than at the initial stage of porous material forming.

(8)When the reduction ratio increases, the material deformation and the work hardening increase. Thus, the compression force and the hardness will increase while the reduction ratio increases.

\section{Acknowledgment}

The authors want to extend their thanks to the Ministry of Science and Technology. The advice and financial support of MOST are gratefully acknowledged.

\section{References}

1. T. S. Yang, Y. C. Hsu, Journal of Materials Processing Technology, 177, 154 (2006)

2. D. N. Lee, H. S. Kim, Powder Metallurgy 35, 275 (1992)

3. A. Shirizly, J. Tirosh, L. Rubinsky, Journal of Materials Science and Engineering, 249, 55 (1998)

4. E. Doege, A Bagaviev, International Journal of Mechanical Sciences, 39 (10), 1151 (1997)

5. C. C. Huang, J. H. Cheng, Journal of Materials Processing Technology, 44, 489 (2002)

6. R. J. Green, International Journal of Mechanical Sciences, 14, 215 (1972)

7. S. Shima, M. Oyane, International Journal of Mechanical Sciences, 18, 285 (1976)

8. S. M .Doraivelu, H. L. Gegel, J. S. Gunasekera, J. C. Malas, J. T. Morgan, International Journal of Mechanical Sciences, 26, 527 (1984)

9. A. D. M. Dias, L. Schaeer, International Journal of Machining Tools and Manufacture, 33, 577 (1993)

10. A. K. Jha, S. Kumar, International Journal of Machine Tool Design \& Research, 26, 369 (1986)

11. D.K.Nguyen, T. T. Tran, and S. G. Buntara, and V. T. Bui, International Journal of Engineering and Technology Innovation, 6 (3), 173 (2016) 